\title{
Entry effects in the narrow, gas lubricated, step slider bearing
}

\author{
I. Penesis ${ }^{1} \quad$ J. J. Shepherd ${ }^{2}$
}

(Received 31 July 2007; revised 28 December 2007)

\begin{abstract}
Singular perturbation methods are applied to an analysis of the operation of an isothermal gas, step slider bearing of narrow geometry and operating at moderate bearing numbers, when the step discontinuity is located very close to the bearing's leading edge. Approximate expressions are obtained for the pressure field in the lubricating gap and the influence of the nature of the bearing step on this is investigated. These provide a convenient explicit means for examining the performance characteristics of the bearing.
\end{abstract}

\section{Contents}

See http://anziamj.austms.org.au/ojs/index.php/ANZIAMJ/article/view/351 for this article, (c) Austral. Mathematical Soc. 2008. Published January 2, 2008. ISSN 1446-8735 
2 Governing equations

3 Perturbation analysis

C263

4 Discussion

C267

References

C270

\section{Introduction}

In analyzing the isothermal operation of a gas lubricated, slider bearing, we seek the structure of the pressure field within the bearing, which directly influences its load bearing properties. The nonlinearity of the equations modelling the bearing action usually means that numerical methods must be used. However, in circumstances where a small parameter may be identified, perturbation techniques obtain closed form approximations to the pressure and associated quantities. This was the approach adopted by Shepherd and DiPrima [7], where narrow bearing geometry provided an appropriate small parameter. Such geometries are seen in the modelling of the operation of the flying head reader in a computer disc memory by Mitsuya et al. [1, 2]. Subsequent work by Penesis et al. [3, 4, 5, 6] applied this approach to narrow bearings of varying geometries, in particular those displaying a step discontinuity in the bearing gap profile [6]. In all these studies, any discontinuity was located interior to the bearing and well clear of the leading and trailing bearing edges. However, the geometries investigated by Mitsuya et al. $[1,2]$ locate a discontinuity very close to the leading edge. Thus, in the present article we use the approach of Penesis et al. $[3,4,5,6]$ to analyze the operation of a narrow bearing with a step discontinuity very close to the bearing gap entry. 


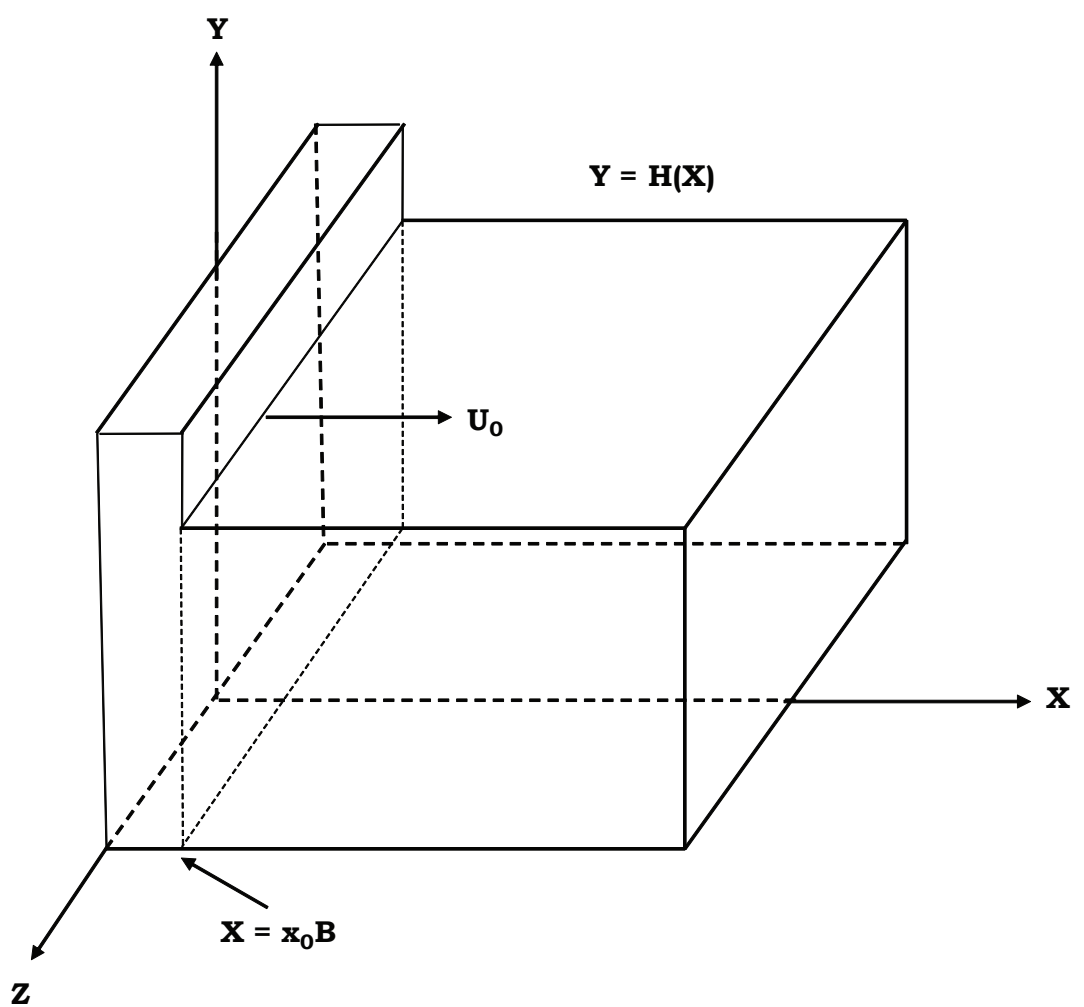

FiguRE 1: Geometry for the narrow gas step slider bearing.

\section{Governing equations}

The geometry of the step slider bearing considered here (of length $L$ and breadth $B$ ) is displayed in Figure 1. The lower surface (the $X Z$ plane here) moves with constant speed $U_{0}$ in the positive $X$-direction, while the (stationary) upper surface represented by $Y=H(X)$ has a single finite transverse jump discontinuity along the line $X=x_{0} B$, for some dimensionless $0<x_{0}<1$. Note that this bearing profile is a wedge; that is, it is independent of $Z$, analogous to that of earlier work by Penesis et al. [6]. 
For the above wedge step, the discontinuous upper planar surface is

$$
H(X)= \begin{cases}H_{0}, & 0 \leq X \leq x_{0} B \\ H_{1}, & x_{0} B \leq X \leq L\end{cases}
$$

for $-B / 2 \leq Z \leq B / 2$ where $H_{0}, H_{1}, x_{0}$ are positive constants, and with $0<x_{0}<1$. Note that $x_{0}$ is dimensionless. Defining $x, z$ and $h$ to be dimensionless versions of $X, Z$ and $H$ scaled against length $L$, breadth $B$ and $H_{0}$ respectively, (1) becomes

$$
h(x, z)= \begin{cases}1, & 0 \leq x \leq \epsilon x_{0} \\ \beta, & \epsilon x_{0} \leq x \leq 1\end{cases}
$$

where $\epsilon=B / L$ is the breadth parameter, and $\beta=H_{1} / H_{0}$. Thus, narrow bearings are characterized by small values of $\epsilon$.

For the case of isothermal flow, the pressure in the bearing gap may be shown to be a solution of the non-linear Reynolds equation $[8,9]$ which is, in dimensionless form,

$$
\epsilon^{2} \frac{\partial}{\partial x}\left(h^{3} p \frac{\partial p}{\partial x}\right)+\frac{\partial}{\partial z}\left(h^{3} p \frac{\partial p}{\partial z}\right)=\epsilon^{2} \Lambda \frac{\partial}{\partial x}(p h),
$$

where $x, z$ and $h$ are as above, and $p$ is the dimensionless pressure, scaled against the (constant) ambient pressure, $P_{a}$. The dimensionless parameter $\Lambda=6 \mu U_{0} L / P_{a} H_{0}^{2}$, is the bearing number, with $\mu$ the viscosity of the gas lubricant. Note that $\Lambda$ is proportional to $U_{0}$, the bearing speed. Note also that (3) does not involve a variable corresponding to $Y$, so the pressure is independent of this variable. This arises from the assumption that the gap between the bearing surfaces is very small relative to the overall bearing dimensions $[8,9]$.

Technically, the pressure $p$ in (3) depends on all of $x, z, \Lambda$ and $\epsilon$. However, since we here apply a perturbation analysis based on $\epsilon \rightarrow 0$ (a narrow bearing) operating at moderate speeds ( $\Lambda$ fixed with $\Lambda=O(1)$ ), we will not display this $\Lambda$-dependence explicitly, and simply write the pressure $p$ as $p(x, z, \epsilon)$. 
The differential equation (3) holds on $0 \leq x \leq 1,-1 / 2 \leq z \leq 1 / 2$, while the condition that the pressure attains the ambient value at the bearing edges becomes

$$
p(x, \pm 1 / 2, \epsilon)=p(0, z, \epsilon)=p(1, z, \epsilon)=1 .
$$

We view the bearing surface as consisting of the union of two smooth bearing surfaces defined on the separate domains

$$
0 \leq x \leq \epsilon x_{0},-1 / 2 \leq z \leq 1 / 2 \text { and } \epsilon x_{0} \leq x \leq 1,-1 / 2 \leq z \leq 1 / 2,
$$

and apply basic techniques [7] separately to these smooth sub-domains. This requires solving (3) subject to appropriate boundary conditions at the edges of these sub-domains. While (4) holds at the exterior boundary of both regions, further conditions along the common boundary at $x=x_{0} \epsilon$ are required. Continuity of $p(x, z, \epsilon)$ there is written as

$$
p\left(\epsilon x_{0}-, z, \epsilon\right)=p\left(\epsilon x_{0}+, z, \epsilon\right)=\lambda(z, \epsilon),
$$

for all $\epsilon>0,-1 / 2 \leq z \leq 1 / 2$, where $\lambda(z, \epsilon)$ is a function to be determined. A further condition arises from the requirement that the mass flow across $x=x_{0} \epsilon$ be continuous, and becomes

$$
h^{3}(x) \frac{\partial p}{\partial x}-\left.\Lambda h(x)\right|_{x=\epsilon x_{0}-}=h^{3}(x) \frac{\partial p}{\partial x}-\left.\Lambda h(x)\right|_{x=\epsilon x_{0}+} .
$$

Thus, on the first domain, this pressure $p(x, z, \epsilon)$ is assumed to satisfy (3), together with

$$
\left.\begin{array}{rl}
p(x, \pm 1 / 2, \epsilon) & =1, \quad 0 \leq x \leq \epsilon x_{0} \\
p(0, z, \epsilon) & =1 \\
p\left(x_{0} \epsilon, z, \epsilon\right) & =\lambda(z, \epsilon)
\end{array}\right\} \quad-1 / 2 \leq z \leq 1 / 2 ;
$$

while on the second it satisfies (3), plus

$$
p(x, \pm 1 / 2, \epsilon)=1, \quad \epsilon x_{0} \leq x \leq 1,
$$




$$
\left.\begin{array}{ll}
p\left(x_{0} \epsilon, z, \epsilon\right) & =\lambda(z, \epsilon) \\
p(1, z, \epsilon) & =1
\end{array}\right\} \quad-1 / 2 \leq z \leq 1 / 2
$$

In the following Section, we apply perturbation methods based on $\epsilon \rightarrow 0$ to obtain representations for the pressure field in the leading and trailing sections of the bearing; and then, by applying the juncture conditions (5) and (6), construct the function $\lambda(z, \epsilon)$.

Compare the present case to that of Penesis et al. [5]. There, the discontinuity was located at $x=x_{0}$, where $0<x_{0}<1$, and $x_{0}$ was independent of $\epsilon$. The subsequent analysis showed that the pressure displayed regions of rapid change (layers) of thickness $O(\epsilon)$ adjacent to the leading and trailing edges $x=0,1$ and on both sides of the discontinuity $x=x_{0}$. Away from the layers, the pressure varied relatively slowly. In the special case of the Rayleigh step (where $h$ is constant in the leading and trailing regions), the layers at $x=0,1$ vanished, and layers only occurred at the discontinuity; while the pressure away from $x=x_{0}$ took on the ambient value $p=1$.

In the present case, the leading bearing section is of length $O(\epsilon)$. We regard this as lying entirely within the layer region adjacent to $x=0$. Thus, all variation of $h$ (including the discontinuity at $x=\epsilon x_{0}$ ) lies within the leading layer. Away from this layer, $h=\beta$, a constant, so by analogy with the Rayleigh step (see above), we expect $p \equiv 1$ throughout this region.

Thus, the solution away from the boundary layer at $x=0$ is

$$
p(x, z, \epsilon)=p_{0}(x, z) \equiv 1,
$$

and is valid even into the layer region.

\section{$3 \quad$ Perturbation analysis}

To analyze the pressure in the leading edge layer incorporating $0 \leq x \leq \epsilon x_{0}$, we introduce the local variable $\xi$, defined by $x=\epsilon \xi$ where $\xi=O(1)$ as $\epsilon \rightarrow 0$. 
Making a change in variable $p(\epsilon x, z, \epsilon) \equiv P(\xi, z, \epsilon)$, our governing differential equation (3) becomes

$$
\frac{\partial}{\partial \xi}\left(h^{3} P \frac{\partial P}{\partial \xi}\right)+\frac{\partial}{\partial z}\left(h^{3} P \frac{\partial P}{\partial z}\right)=\epsilon \Lambda \frac{\partial}{\partial \xi}(P h) .
$$

From (11), $p_{0}(x, z) \equiv 1$ away from the layer region, hence we seek a boundary layer expansion in the region $0 \leq x \leq \epsilon x_{0}$ of the form

$$
P(\xi, z, \epsilon)=1+\epsilon P_{1}(\xi, z)+\epsilon^{2} P_{2}(\xi, z)+\cdots .
$$

Since $\lambda(z, \epsilon)=P\left(x_{0}, z, \epsilon\right)$, (13) must converge to this value. We thus propose

$$
\lambda(z, \epsilon)=1+\epsilon \lambda_{1}(z)+\epsilon^{2} \lambda_{2}(z)+\cdots,
$$

where $\lambda_{1}(z), \lambda_{2}(z), \ldots$ are to be determined.

Substituting expressions (2), (13) and (14) into (12) and equating like powers of $\epsilon$ gives the differential equation for $P_{1}$ as

$$
\frac{\partial}{\partial \xi}\left(\frac{\partial P_{1}}{\partial \xi}\right)+\frac{\partial}{\partial z}\left(\frac{\partial P_{1}}{\partial z}\right)=0
$$

to be solved subject to the boundary conditions

$$
P_{1}(0, z)=0, \quad P_{1}\left(x_{0}, z\right)=\lambda_{1}(z), \quad P_{1}(\xi, \pm 1 / 2)=0 .
$$

This gives

$$
P_{1}(\xi, z)=\sum_{n=1}^{\infty} c_{n} \phi_{n}(z) \sinh n \pi \xi, \quad-1 / 2<z<1 / 2, \quad \xi \geq 0,
$$

where

$$
\phi_{n}(z)= \begin{cases}\cos (n \pi z), & n=1,3,5, \ldots \\ \sin (n \pi z), & n=2,4,6, \ldots\end{cases}
$$


and

$$
c_{n}=\frac{1}{\sinh n \pi x_{0}} \int_{-1 / 2}^{1 / 2} \lambda_{1}(z) \phi_{n}(z) d z, \quad n=1,2,3, \ldots .
$$

Hence, the uniform expansion for the pressure takes the form

$$
p(x, z, \epsilon)=1+\epsilon P_{1}(\xi, z)+O\left(\epsilon^{2}\right)
$$

on the domain $0 \leq x \leq \epsilon x_{0},-1 / 2 \leq z \leq 1 / 2$.

Consider now the region $x \geq \epsilon x_{0}$ that lies within the $O(\epsilon)$ layer at $x=0$. Assuming the same local variable $\xi(x=\epsilon \xi$ where $\xi=O(1)$ as $\epsilon \rightarrow 0)$ and making a change in variable $p(\epsilon \xi, z, \epsilon) \equiv \tilde{P}(\xi, z, \epsilon)$, our governing differential equation (3) becomes

$$
\frac{\partial}{\partial \xi}\left(h^{3} \tilde{P} \frac{\partial \tilde{P}}{\partial \xi}\right)+\frac{\partial}{\partial z}\left(h^{3} \tilde{P} \frac{\partial \tilde{P}}{\partial z}\right)=\epsilon \Lambda \frac{\partial}{\partial \xi}(\tilde{P} h) .
$$

We assume the boundary layer expansion for $x \geq \epsilon x_{0}$ takes the form

$$
\tilde{P}(\xi, z, \epsilon)=1+\epsilon \tilde{P}_{1}(\xi, z)+\epsilon^{2} \tilde{P}_{2}(\xi, z)+\cdots .
$$

Substituting expressions (2), (14) and (22) into (21) and equating like powers of $\epsilon$ gives the boundary value problem for $\tilde{P}_{1}$ as

$$
\begin{aligned}
& \frac{\partial}{\partial \xi}\left(\frac{\partial \tilde{P}_{1}}{\partial \xi}\right)+\frac{\partial}{\partial z}\left(\frac{\partial \tilde{P}_{1}}{\partial z}\right)=0, \\
& \tilde{P}_{1}\left(x_{0}, z\right)=\lambda_{1}(z), \quad \tilde{P}_{1}(\infty, z)=0, \quad \tilde{P}_{1}(\xi, \pm 1 / 2)=0 .
\end{aligned}
$$

The second of (24) is a matching condition, arising from the requirement that the expansion (22) should match the solution (11) as we exit the layer at $x=0$, that is, as $\xi \rightarrow \infty$.

The solution of the above boundary value problem is

$$
\tilde{P}_{1}(\xi, z)=\sum_{n=1}^{\infty} d_{n} \phi_{n}(z) e^{-n \pi \xi}, \quad-1 / 2<z<1 / 2, \quad \xi \geq 0,
$$


where

$$
d_{n}=e^{n \pi x_{0}} \int_{-1 / 2}^{1 / 2} \lambda_{1}(z) \phi_{n}(z) d z, \quad n=1,2,3, \ldots
$$

and the $\phi_{n}(z)$ are as in (18).

Hence, the uniform pressure takes the form

$$
p(x, z, \epsilon)=1+\epsilon \tilde{P}_{1}(\xi, z)+O\left(\epsilon^{2}\right),
$$

on the region $x \geq \epsilon x_{0}$ and on to the 'outer' region where the pressure takes the ambient value $p \equiv 1$.

Expressions (20) and (27) with (17) for $P_{1}$ and (25) for $\tilde{P}_{1}$, provide a straightforward procedure for calculating the pressure distribution in a step slider bearing. However, to determine these quantities, $\lambda_{1}$ needs to be found.

The pressure, (20) and (27) in the layer section of the bearing is continuous at the step $x=\epsilon x_{0}$, taking the value $\lambda_{1}$ there. To determine $\lambda_{1}$, the mass flow condition (6) is invoked. Substituting (20) and (27) into condition (6) and equating like terms, we obtain

$$
\left.\epsilon \frac{\partial P_{1}}{\partial x}\right|_{x=\epsilon x_{0}}-\left.\beta^{3} \epsilon \frac{\partial \tilde{P}_{1}}{\partial x}\right|_{x=\epsilon x_{0}}=\Lambda(1-\beta) .
$$

On substituting for $P_{1}(\xi, z)$ and $\tilde{P}_{1}(\xi, z)$ and equating like powers of $\epsilon$ we obtain

$$
\sum_{n=1}^{\infty} n \pi \phi_{n}(z)\left(c_{n} \cosh n \pi x_{0}+d_{n} \beta^{3} e^{-n \pi x_{0}}\right)=\Lambda(1-\beta) .
$$

Consider now the expression (29). The right-hand side is a constant, hence an even function in $z$; so that from the form of the eigenfunctions $\phi_{n}(z)$, we deduce

$$
c_{n}=d_{n}=0, \quad n=2,4,6, \ldots
$$


For $n=1,3,5, \ldots,(29)$ becomes

$$
\sum_{n=1,3,5, \ldots}^{\infty} n \pi\left[\operatorname{sech}\left(n \pi x_{0}\right)+\beta^{3}\right]\left\{\int_{-\frac{1}{2}}^{\frac{1}{2}} \lambda_{1}(z) \cos (n \pi z) d z\right\} \cos (n \pi z)=\Lambda(1-\beta),
$$

from which we obtain a Fourier representation for $\lambda_{1}(z)$ as

$$
\lambda_{1}(z)=\frac{2 \Lambda(1-\beta)}{\pi^{2}} \sum_{n=1,3,5, \ldots}^{\infty} \frac{\sin (n \pi / 2)}{n^{2}\left[\operatorname{sech}\left(n \pi x_{0}\right)+\beta^{3}\right]} \cos n \pi z,
$$

giving the $O(\epsilon)$ term in the expansion (14) for $\lambda(z, \epsilon)$.

Combining the findings of the previous section, we find that the pressure distribution over the whole bearing domain $0 \leq x \leq 1,-1 / 2 \leq z \leq 1 / 2$ is given approximately by

$$
\begin{aligned}
p(x, z, \epsilon)=1+ & \frac{\epsilon \Lambda(1-\beta)}{\pi^{2}} \sum_{m=0}^{\infty} G_{m} \operatorname{cosech}\left((2 m+1) \pi x_{0}\right) \times \\
& \times \sinh ((2 m+1) \pi x / \epsilon) \cos ((2 m+1) \pi z)
\end{aligned}
$$

on $0 \leq x \leq \epsilon x_{0}$, and

$$
p(x, z, \epsilon)=1+\frac{\epsilon \Lambda(1-\beta)}{\pi^{2}} \sum_{m=0}^{\infty} G_{m} e^{(2 m+1) \pi\left(x_{0}-x / \epsilon\right)} \cos [(2 m+1) \pi z],
$$

on $\epsilon x_{0} \leq x \leq 1$ where

$$
G_{m}=\frac{(-1)^{m}}{(2 m+1)^{2}\left[\operatorname{sech}\left((2 m+1) \pi x_{0}\right)+\beta^{3}\right]} .
$$

\section{Discussion}

For a given step as described by (2), the expansions (33) and (34) define an explicit and useful approximation to the pressure field throughout the 


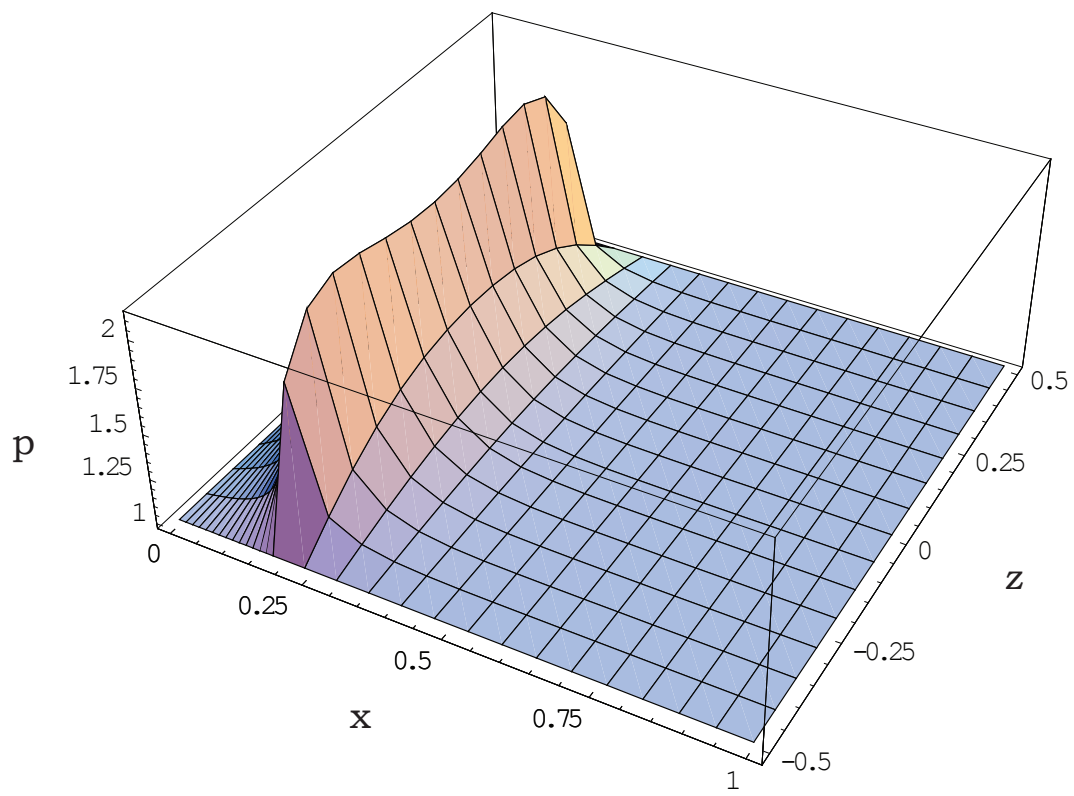

Figure 2: Surface plot of non-dimensional pressure for the wedge step slider bearing as given by asymptotic formulae (33) and (34) for the linear bearing profile (36), with $\epsilon=0.2, \Lambda=20$ and $x_{0}=0.9$.

bearing, valid over an appropriate range of $\epsilon$ and $\Lambda$ values. They show that the pressure is within $O(\epsilon)$ of the ambient value for $x=O(\epsilon)$, but for $x$ bounded away from zero as $\epsilon \rightarrow 0$, the pressure is effectively the ambient. This is as anticipated. Note also that when $\beta=1$, corresponding to $h(x) \equiv 1$, the pressure reduces to $p=1$ everywhere, again as expected.

Figure 2 displays the (approximate) three dimensional pressure distribution as given by (33) and (34) for a simple Rayleigh bearing profile

$$
h(x, z)= \begin{cases}1, & 0 \leq x \leq 0.18 \\ 0.4, & 0.18 \leq x \leq 1\end{cases}
$$

for which $x_{0}=0.9$ and $\epsilon=0.2$. Figure 3 displays the pressure as given by 


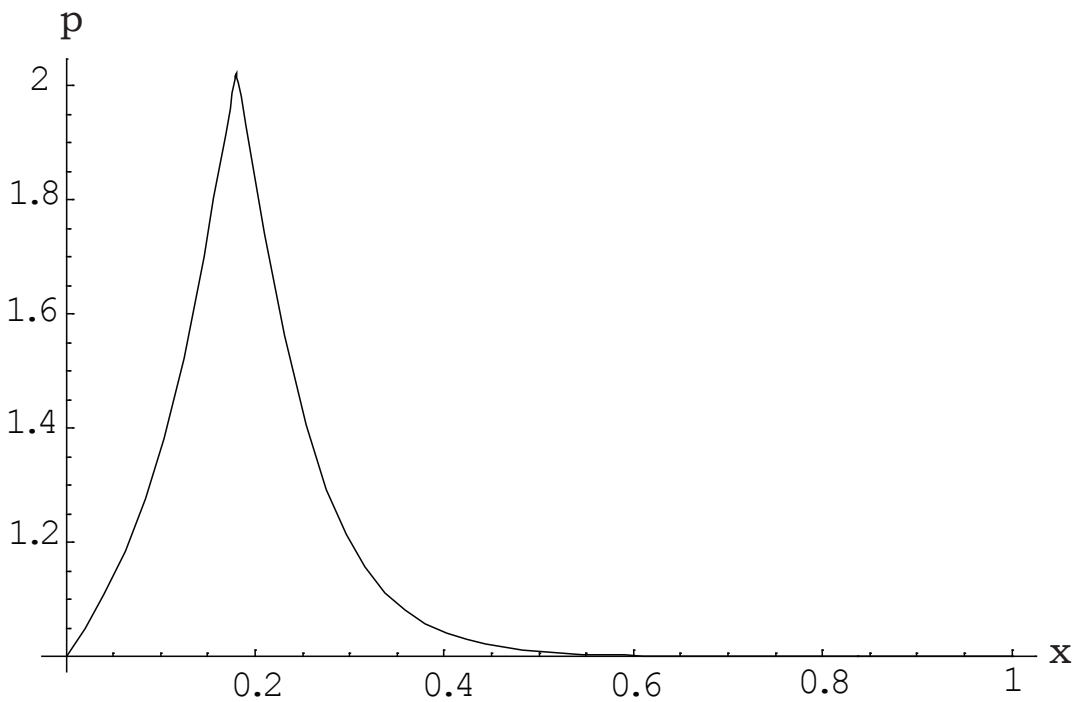

Figure 3: One-dimensional plot of the pressure for the wedge step bearing along $z=0$ as given by asymptotic formulae (33) and (34) for the linear bearing profile (36), with $\epsilon=0.2, \Lambda=20$ and $x_{0}=0.9$. 
(33) and (34), along the bearing midline $z=0$. In both cases, the layer at the step $x=x_{0} \epsilon=0.18$ is clearly in evidence, with the pressure rising rapidly from ambient to about twice that value, then decaying equally rapidly back to ambient. Note that the pressure, while continuous at $x=0.18$, shows a slope discontinuity there, arising from the imposition of the mass flow condition (6). These results agree, at least qualitatively, with those obtained numerically by Mitsuya [1] for a comparable (but not identical) situation. There, the pressure showed a steep entry layer, followed by slow variation past the discontinuity point, arising from a non-constant bearing profile there. While the analysis here could be adapted to deal with that situation, this is beyond the scope of the present paper. Our results also show that the load carrying capacity of the bearing (effectively the integral over the bearing of the excess of pressure above the ambient) derives almost completely from the region around the step discontinuity, causing the centre of pressure (the areal moment of the excess pressure) to be located in this small entry region.

\section{References}

[1] Mitsuya, Y. and Ohkubo, T., High Knudsen number molecular rarefaction effects in gas-lubricated slider bearings for computer flying heads, J. Tribology, Trans. ASME, 109, April 1987, pp.276-282. C259, $\mathrm{C} 270$

[2] Mitsuya, Y., Deng, Z., Ohka, M., Derivation of rarefaction-modified Reynolds equation considering porosity of thin lubricant film, $J$. Tribology, Trans. ASME, 119, No. 4, 1997, pp.653-659. C259

[3] Penesis, I., Shepherd,J. J., and Connell, H. J., Asymptotic analysis of narrow gas-lubricated slider bearings with non-smooth profiles, Proceedings of EMAC98, 3rd Biennial Engineering Mathematics and Applications Conference, The Institution of Engineers., Adelaide, Australia, 1998, pp.163-166. C259 
[4] Penesis, I., Shepherd,J. J., and Connell, H. J., The pressure field in a two-dimensional taper-taper gas-lubricated bearing of narrow geometry, EMAC 2000 Proceedings, 3rd Biennial Engineering Mathematics and Applications Conference, The Institution of Engineers., Melbourne, Australia, 2000, pp.239-242. C259

[5] Penesis, I., Shepherd, J. J. and Connell, H. J., The pressure field in the gas-lubricated step-slider bearing of narrow geometry, ANZIAM J., 45(3), January, 2004, pp.423-442.

http://www. austms.org. au/Publ/ANZIAM/V45P3/2014.html. C259, C263

[6] Penesis, I., and Shepherd, J. J., The gas-lubricated Rayleigh step slider bearing of narrow geometry, ICIAM 2003, 5th International Congress on Industrial and Applied Mathematics, EMAC 2003 Proceedings, Sydney, Australia, July 2003, pp.187-191. C259, C260

[7] Shepherd, J. J., and DiPrima, R. C., Asymptotic analysis of a finite gas slider bearing of narrow geometry, J. Lub. Tech., Trans. ASME, 105, 1983, pp.491-495. C259, C262

[8] Gross, W. A., Matsch, L. E., Castelli, V., Eshel, A., Vohr, J. H., and Wildman.M., Fluid Film Lubrication, Wiley, New York, 1980. C261

[9] Dowson, D., A Generalized Reynolds Equation for Fluid-Film Lubrication, Int. J. Mech Sci., 4, 1962, pp.159-170. C261 


\section{Author addresses}

1. I. Penesis, Dept. Maritime Engineering, Australian Maritime College, Launceston, Australia.

mailto:i.penesis@amc.edu. au

2. J. J. Shepherd, School of Mathematical \& Geospatial Sciences, RMIT University, Melbourne, Australia.

mailto: jshep@rmit.edu.au 